Rückblickend betrachtete Kennan seine Tätigkeit in der Moskauer Botschaft unter der Verantwortung von Bullitt und der Leitung des Geschäftsträgers Loy Henderson der in einem Brief an Robert Kelley im April 1937 das gesellschaftlich-atmosphärische Klima der Verfolgung zur Zeit des Massenterrors in die eindrücklichen Worte kleidete, dass »keiner [...] zu wissen [scheint], wer als nächster verschwindet ${ }^{51}-$ als überaus lehrreiche Jahre, insofern sie

die ersten [waren], die ihre Arbeiten intellektuell, ja wissenschaftlich angingen. Wir waren bestrebt, unsere politische Berichterstattung nach denselben Grundsätzen der Objektivität und wissenschaftlichen Genauigkeit auszurichten wie seinerzeit die Arbeit in Riga. Wir waren zutiefst von der Wichtigkeit der sowjetisch-amerikanischen Beziehungen für Gegenwart und Zukunft [überzeugt]. Dabei hielten wir es für entscheidend, das Wesen des sowjetischen Regimes korrekt zu analysieren und gangbare Wege für Verhandlungen zu erforschen. ${ }^{52}$

\title{
1.5 Die Konfrontation mit dem Nationalsozialismus und dem Sowjetkommunismus während des Zweiten Weltkrieges und die Rolle von Intellektuellen und Wissenschaftlern in der Abteilung "Research and Analysis" des US-amerikanischen Geheimdienstes
}

Überlegungen seitens der US-amerikanischen Regierungsstellen über die gesamteuropäische Nachkriegsordnung nach dem Ende des Zweiten Weltkrieges setzten alsbald nach dem Entschluss ein, den militärischen Kampf gegen das nationalsozialistische Herrschaftssystem aufzunehmen. Hierbei geriet auch die Frage in den Fo-

51 Zit. n. ebd., S. 37.

52 Kennan, Memoiren eines Diplomaten, S. 87 f. Wie tief Kennan durch seine Moskauer Lehrjahre geprägt wurde und mithin eine entscheidende Rolle bei der zukünftigen Bewertung und Einschätzung der diplomatischen Beziehungen zwischen den Vereinigten Staaten und der Sowjetunion spielte, zeigte eine schriftliche Ausarbeitung von ihm aus dem Jahre 1936 respektive 1937, aus der hervorging, wie realistisch er die stalinsche Außenpolitik im Kontext des sogenannten voreuropäischen Krieges, d. h. des Zweiten Weltkrieges, beurteilte. In dem nicht für offizielle US-amerikanische Stellen gedachten persönlichen Arbeitspapier »Das Kriegsproblem in der Sicht der Sowjetunion« aus der Mitte der 1930er-Jahre versuchte Kennan, die zukünftige sowjetische Außenpolitik unter Stalin zu prognostizieren. Auf der Grundlage eines tief verankerten russischen Chauvinismus und der Xenophobie begegnete die sowjetische Regierung jedweden Fremden mit dem »Geist des Mißtrauens und des Haßes«, sodass nicht zuletzt deshalb neben genuinen ideologischen Gegensätzen alle nichtkommunistischen Staaten für die Sowjetunion per definitionem politische Feinde waren, die es galt mit allen Mitteln zu bekämpfen. Ebendies schloß nach Auffassung von Kennan nicht aus, dass Stalin geschickt auf der Klaviatur der außenpolitischen Instrumente zu spielen sich imstande zeigte und bereit war, mit anderen Staaten Nichtangriffs- und Beistandspakte abzuschließen. Für Kennan folgte daraus zum damaligen Zeitpunkt, dass Stalin möglicherweise auch bereit wäre, mit dem deutschen Nationalsozialismus auf staatlicher Ebene zu kooperieren, um einerseits in dem bevorstehenden europäischen Krieg sich zu einem frühen Zeitpunkt heraushalten zu können und andererseits sich später sich als sogenannter Aasgeier beteiligen zu können. Im Nachhinein enthielt für Kennan das persönliche Arbeitspapier die Vorhersage für den nazistisch-sowjetischen Nichtangriffspakt, die eine für ihn genaue Beurteilung der sowjetrussischen Außenpolitik enthielt, allerdings auch eine erhebliche Unterschätzung der gesamteuropäischen Gefahren durch die hitlerschen »pangermanischen «Expansionsziele. Siehe hierzu die Ausführungen von Kennan, in: Ebd., S. 76-79. 
kus des Interesses, wie künftig die außenpolitische Beziehung mit dem zweifelsohne kriegsstrategisch wichtigsten und militärisch stärksten Partner in der Anti-Hitler-Koalition, der Sowjetunion, sich gestalten könnte und ob trotz der systemimmanenten Widersprüche ein modus vivendi mit Stalin denkbar wäre. Um das nach der deutschen Kriegserklärung an die Vereinigten Staaten im Dezember 1941 resultierende Primärziel - dem alles andere untergeordnet wurde - zu erreichen, den als weltweite fundamentale Gefahr erkannten »braunen« Totalitarismus zu besiegen, wurde in Washington eine Vielzahl von Diensten und Apparaten geschaffen, die die notwendigen außen- und militärpolitischen Entscheidungsprozesse von Roosevelt vorbereiten sollten; nach Beendigung des Krieges wurde deren Ausbau vorangetrieben, um in der sich herauskristallisierenden Auseinandersetzung mit dem "roten« Totalitarismus sowjetischer Couleur bestmöglichst vorbereitet zu sein.

Um indessen die Politik Washingtons in der Zeit der politischen, militärischen, kulturellen sowie ideologischen Konfrontation mit der Sowjetunion als Zentrum der europäischen kommunistischen Staatenwelt angemessen einschätzen und bewerten zu können, ist ein Blick auf den Zweiten Weltkrieg vonnöten. Frühe Überlegungen und Planungen über die Nachkriegspolitik der Vereinigten Staaten setzten, wie gesagt, bereits im Verlauf der konkreten kriegerischen Konfrontation mit dem existenziellen politischen Regimegegner aus Deutschland ein. Ein Ausdruck dieser weitsichtigen Politik in Washington war die Schaffung der Coordinator of Information (COI), des ersten staatlichen Geheimdienstapparates der Vereinigten Staaten, dessen Gründung durch Roosevelt bereits wenige Wochen nach dem Überfall der deutschen Wehrmacht auf die Sowjetunion am 11. Juli 1941 beschlossen wurde. Als treibende Kraft im Hintergrund der staatlichen Machtinstitutionen fungierte der renommierte New Yorker Rechtsanwalt William J. Donovan, der seine Beziehungen und Einflüsse nutzte und letzten Endes Roosevelt aufgrund der absolut angespannten weltpolitischen Kontextbedingungen davon überzeugen konnte, den amerikanischen Geheimdienstapparat zu gründen, als dessen Direktor er vom Präsidenten berufen wurde. Donovan konzipierte die COI höchstpersönlich und besorgte sich teilweise schon vor dem Eintritt der USA in den Zweiten Weltkrieg - am 11. Dezember 1941 erklärte Hitler den Vereinigten Staaten offiziell den Krieg - größtenteils nach persönlichem Gutdünken sein ihm direkt unterstelltes und zuarbeitendes Mitarbeiterteam. $\mathrm{Zu}$ den zentralen Aufgaben des Geheim- und Nachrichtendienstes gehörte von Anfang an, »auf umfassender Sachkenntnis beruhende Studien und Analysen zu aktuellen politischen und militärischen Fragen auszuarbeiten. Donovan war der Ansicht, daß für diese Aufgabe die besten Geistes- und Sozialwissenschaftler rekrutiert werden sollten. « $^{53}$

Genau diese Tatsache, dass nämlich seit 1942 unter anderem bekannte amerikanische Wissenschaftler, Persönlichkeiten und Intellektuelle aus den verschiedensten gesellschaftlichen Bereichen aktiv einen Beitrag innerhalb des US-Geheimdienstes

53 Petra Marquardt-Bigman, Amerikanische Geheimdienstanalysen über Deutschland 1942-1949, München 1995, S. 7. Diese sehr gute Studie über die »höchst unorthodoxe Organisation « und den komplizierten Aufbau, die Aufgabenbereiche und des konkreten Einflusses des OSS sowie der R\&A im Kontext des Apparatdschungels der schwer überschaubaren Washingtoner Administration vor allem im Zweiten Weltkrieg ist nicht zuletzt aufgrund ihres empirischen Gehalts - die Studie beruht vor allem auf dem umfangreichen Aktenmaterial des OSS - und ihrer differenzierten Einschätzung überaus lesenswert. Im deutschsprachigen Raum ist diese Untersuchung die umfangreichste und wichtigste Veröffentlichung zu diesem Thema. 
leisteten, um den scheinbar nicht aufzuhaltenden, von Erfolg zu Erfolg eilenden und sich ständig radikalisierenden deutschen Machtapparat aufzuhalten und zu besiegen, machte die neugegründete COI zu einem institutionellen Phänomen sui generis. Innerhalb des COI-Apparates wiederum war es die alsbald ins Leben gerufene Abteilung R\&A, die sich sehr schnell einen Namen machte und innerhalb der US-Administration große Anerkennung fand. Ausdruck dieses weitverbreiteten Respektes - wiewohl es freilich auch viele Neider gab -, den man dieser bemerkenswerten Abteilung gegenüber hatte, war die Erfindung des aussagekräftigen Spitznamens "The Campus«. Denn durch die geschickte Personalpolitik des ab Sommer 1942 als Leiter dieser Abteilung fungierenden und an der Harvard University lehrenden Historikers William L. Langer ${ }^{54}$ arbeiteten bereits nach wenigen Monaten bekannte und bedeutende Historiker, Politikwissenschaftler, Soziologen, Wirtschaftswissenschaftler, Psychologen, Geografen und Sprachwissenschaftler von sehr vielen Universitäten der Vereinigten Staaten für die R\&A - die nach regionalen Bereichen untergliedert war und unterteilt wurde in die vier R\&A-Divisionen Europa-Afrika, UdSSR, Fernost und Lateinamerika. Diese vier Divisionen waren noch einmal in sich funktional und regional untergliedert. Somit entstand im Kampf gegen das nationalsozialistische Herrschaftssystem beispielsweise auch noch ab Anfang des Jahres 1943 die schätzungsweise 40 Personen umfassende Central European Section (CES) der R\&A im OSS, die in allererster Linie für Deutschland zuständig war und in der die bereits zu diesem Zeitpunkt bekannten deutschen Emigranten, Wissenschaftler und Intellektuellen eingesetzt wurden, die zum Teil an dem berühmten, wenige Wochen nach der politischen Machtübertragung der Nationalsozialisten und der Ernennung Hitlers zum Reichskanzler im Januar 1933 letztendlich nach New York geflüchteten Frankfurter Institut für Sozialforschung um den Leiter Max Horkheimer gearbeitet hatten. Genannt werden muss insbesondere der damals bedeutendste NS-Forscher Franz Leopold Neumann, der 1942 mit seinem Buch Behemoth ${ }^{55}$, wie gesagt, eine bahnbrechende Studie über den National-

$54 \mathrm{Vgl}$. die Autobiografie William L. Langer, In and Out of the Ivory Tower. The Autobiography of William L. Langer, New York 1977. Langer wurde 1942 Nachfolger des Historikers James Phinney Baxter als Leiter der R\&A. Als Sohn deutscher Einwanderer interessierte ihn früh die Geschichte. Nach der Promotion lehrte er an der Clark University in Worcester europäische Ceschichte und begann mit seiner Mitarbeit an der Zeitschrift Foreign Affairs, die zu einer der einflussreichsten und wichtigsten Zeitschriftenorgane der Vereinigten Staaten reüssieren sollte. Wichtig ist insbesondere die Tatsache, dass sich Langer schon in den 1930er-Jahren mit der politischen und geschichtlichen Entwicklung Deutschlands beschäftigte. Dabei entstand 1938 der Artikel When Cerman Dreams Come True, in der er das einflussreiche Buch Hitlers Mein Kampfauf seine nationalistischen, sozialdarwinistischen und imperialistischen Implikationen hin analysierte. Zentrale Bedeutung hatte dieser Artikel auch, weil Langer grosso modo die Arbeitsweise der R\&A bereits im Jahre 1938 vorwegnimmt. Siehe hierzu Marquardt-Bigman, Amerikanische Geheimdienstanalysen über Deutschland 1942-1949, S. 28 f.

55 Franz Neumann, Behemoth. The Structure and Practice of National Socialism 1933-1944, New York 1942 (erweit. Ausg. 1944; dt. Ausg. 1977 unter dem Titel Behemoth. Struktur und Praxis des Nationalsozialismus 1933-1944). Erinnert sei an dieser Stelle, dass ein weiterer Emigrant aus Deutschland, nämlich der aus Berlin 1938 in die USA geflüchtete Ernst Fraenkel, bereits 1941 seine berühmte Untersuchung The Dual State über den Nationalsozialismus veröffentlichte, die 1974 auf Deutsch erschien. Von Marcuse stammen mehrere Arbeiten zum >Dritten Reich. Im Kontext seiner Tätigkeit für den OSS erstellte er verschiedene Aufsätze, in denen er das Phänomen sui generis, den Nationalsozialismus und den psychologischen, politischen, ideologischen sowie kulturellen «Kitt zwischen Führung und deutscher Bevölkerung beleuchtete. Vgl. hierzu Herbert Marcuse, Feindanalysen. Über die Deut- 
sozialismus veröffentlichte; zudem Herbert Marcuse, Otto Kirchheimer, Arkadij Gurland, John H. Herz und Felix Gilbert, die nach Kriegsende zur Crème de la Crème der amerikanischen Sozial-, Politik- und Geschichtswissenschaft aufstiegen. Zu diesem intellektuellen Emigrantenkreis in der R\&A gehörten noch neben Carl Schorske und H. Stuart Hughes unter anderem der Historiker Hajo Holborn, der Politologe Karl Deutsch, der Kunstgeschichtler Richard Krautheimer, der Kinderpsychologe Kurt Lewin, der Wirtschaftswissenschaftler Walter Levy, der Jurist Robert Kempner und der frühere preußische Innenminister Albert Grzesinski. ${ }^{56}$

Die gemeinsame existenzielle Grunderfahrung dieses heterogenen Personenkreises war die von politischer Verfolgung, persönlicher Flucht sowie Vertreibung auch von jüdischen Verwandten, Mitbürgern und Freunden durch das nationalsozialistische Gewaltsystem. Zudem natürlich die zu diesem Zeitpunkt nicht für möglich gehaltenen nationalsozialistischen Massenmorde an den osteuropäischen Juden sowie den im Kontext des nach rassistisch-politischen Gesichtspunkten geführten Weltanschauungskrieges in den von der deutschen Wehrmacht eroberten Gebieten Osteuropas deportierten und in größerem Umfang umgebrachten (jüdischen und) nichtjüdischen Zivilisten. Ab Frühjahr 1942 begann die systematische Vernichtung der europäischen Juden, die in zahlreichen Berichten und veröffentlichten wissenschaftlichen Aufsätzen von insbesondere russischen und polnischen jüdischen Flüchtlingen an- und besprochen wurde, sodass speziell die amerikanische Öffentlichkeit frühzeitig unterrichtet werden konnte ${ }^{57}$ (hierbei sind Parallelen zur sogenannten Sowjetforschung zu

schen. Hg. von Peter-Erwin Jansen, Lüneburg 1998; Peter-Erwin Jansen u. a. (Hg.), Zwischen Hoffnung und Notwendigkeit. Texte zu Herbert Marcuse, Frankfurt a. M. 1999, speziell im Vorwort die S. 7-10; vgl. auch Herbert Marcuse, Reason and Revolution. Hegel and the Rise of Social Theory, New York 1941 (dt. Ausg.: Vernunft und Revolution. Hegel und die Entstehung der Cesellschaftstheorie, Neuwied/Berlin 1962). - In den späten 1930er-Jahren erschienen zwei Bücher, die für die zukünftige NSForschung und die Analyse des totalitären Herrschaftsregimes Hitlers durchaus überragende Bedeutung hatten. Der frühere Anhänger Hitlers und der NSDAP Hermann Rauschning veröffentlichte nach seiner Emigration in die USA 1936 zwei Bücher: zum einen Die Revolution des Nihilismus (Zürich 1938) und zum anderen Gespräche mit Hitler (Zürich 1940). Erst mehrere Jahrzehnte später konnte aufgrund der neueren Forschung zum Nationalsozialismus in den 1990er-Jahren nachgewiesen werden, dass diese Gespräche mit Hitler in dieser Form nicht stattgefunden haben können. Eine weithin rezipierte Lizenzausgabe erschien in den USA bei der Alliance Book Corporation in New York bei dem in Deutschland geborenen Verleger Heinz Günther Koppell. Wichtig ist an dieser Stelle die Tatsache, dass in diesem Buchverlag, neben dem Rauschning-Band zum Nationalsozialismus, im selben Jahr u. a. das an anderer Stelle erwähnte Renegatenbuch Out of the Night veröffentlicht wurde.

56 Vgl. zum Personenkreis, den Aktivitäten und Forschungsanalysen speziell der deutschen Emigranten in der Abteilung R\&A für den Zeitraum von 1943 bis 1945 sowie von 1946 bis 1949 die Untersuchung von Alfons Söllner (Hg.), Zur Archäologie der Demokratie in Deutschland, Frankfurt a. M. 1986, Bd. 1: Analysen von politischen Emigranten im amerikanischen Geheimdienst 1943-1945, Bd. 2: Analysen von politischen Emigranten im amerikanischen Außenministerium 1946-1949; vgl. darüber hinaus vor allem die Standardwerke zum OSS von Barry M. Katz, Foreign Intelligence. Research and Analysis in the Office of Strategic Services 1942-1945, London 1989; Bradley F. Smith, The Shadow Warriors. OSS and the Origins of the CIA, New York 1983; Richard Harris Smith, OSS. The Secret History of America's First Central Intelligence Agency, Berkley 1972.

57 Es ist aus heutiger Perspektive immer wieder erstaunlich, wie früh einerseits bahnbrechende seriöse Forschungsliteratur über den Nationalsozialismus erschien. Erinnert sei nochmals an die analytisch herausragenden Veröffentlichungen von Neumann oder Fraenkel aus den frühen 1940er-Jahren. Andererseits lagen frühzeitig Erkenntnisse über die Qualität und den Umfang der nationalsozialis- 
konstatieren, denn wie gezeigt werden konnte, war das Wissen insbesondere in den Vereinigten Staaten über die Sowjetunion und die im dortigen Herrschaftsbereich erfolgten Massenmorde und -verbrechen gerade durch Veröffentlichungen von Renegaten früh vorhanden und weitverbreitet). Unter dem quasi totalitären Schock stehend, den Holocaust so gerade eben noch überlebt zu haben, war den deutschen politischen Emigranten klar und wurde mithin für diesen Lebensabschnitt zur alles andere überschattenden Aufgabe und individuellen Verpflichtung, alles erdenklich Mögliche zu tun, um die deutsche Eroberungs- und Vernichtungsmaschinerie aufzuhalten und den nationalsozialistischen Totalitarismus militärisch, ökonomisch und letzten Endes auch auf der psychologischen Ebene zu besiegen. ${ }^{58}$ Damit sollten die Weichen gestellt werden, damit nach der Niederlage in Deutschland ein funktionierendes kapitalistisches und parlamentarisch-demokratisches System nach amerikanischem und englischem Vorbild aufgebaut werden konnte.

tischen Verbrechen und Verfolgungspolitik vor. Darüber hinaus war auch das Wissen über den Beginn und den Umfang der systematischen Vernichtung der europäischen Juden seit dem Frühjahr 1942 auch und vor allem der amerikanischen Öffentlichkeit verbreitet. Auf den singulären Charakter der deutschen Judenvernichtung insistierte z. B. schon im Januar 1944 Koestler in seinem Beitrag für das New York Times Magazine. Für den Renegaten waren die zu diesem Zeitpunkt der US-Öffentlichkeit bekannten Ausmaße der deutschen Massenmorde an den Juden von drei Millionen Toten »die größte Massentötung in der uns überlieferten Geschichte«. Arthur Koestler, Warum Greueltaten nicht geglaubt werden. Abgedruckt in Koestler, The Yogi and the Commissar, hier zit. n. der dt. Ausg., S. 139. Die Judenpolitik des Nationalsozialismus war seit Anfang 1940 in den USA Gegenstand der Forschung. Im vom »American Jewish Congress « in New York gegründeten jüdischen historischen Institut, dem »Institute of Jewish Affairs«, entwickelte sich eine intensive Auseinandersetzung mit der antisemitischen Politik des NS-Regimes in Europa, die aufgrund der systematischen Materialsammlung zu ersten Veröffentlichungen führte und die ihnen zudem die Chance gab, im Laufe der Jahre die amerikanische Öffentlichkeit über die jüdische Tragödie zu informieren. Hier erschienen auch allein im Zeitraum von 1941 bis 1944 knapp 20 Veröffentlichungen über das Schicksal der Juden im europäischen nationalsozialistischen Unterdrückungssystem. Vgl. zur wissenschaftlichen Erforschung des Holocaust den informativen Aufsatz von Martin Broszat, »Holocaust« und die Ceschichtswissenschaft, in: Vierteljahreshefte für Zeitgeschichte 27 (1979), S. 285-298. Vgl. auch die Literaturhinweise in der frühen Untersuchung Die Endlösung. Hitlers Versuch der Ausrottung der Juden 1939-1945 (Berlin 1956) von dem englischen Historiker Gerald Reitlinger (die engl. Ausgabe erschien 1953 unter dem Titel The Final Solution - The Attempt to Exterminate the Jews of Europe 1939-1945). Das ganz Besondere an der Untersuchung von Reitlinger war vor allem zweierlei: Zum einen stellte sich hier zum ersten mal ein nichtjüdischer Historiker dem extrem schwierigen Unterfangen, den fast geglückten Versuch der deutschen Nationalsozialisten, die gesamte jüdische Bevölkerung Europas systematisch zu ermorden - also die »Endlösung der Judenfrage« zu erreichen - und zum anderen benutzte Reitlinger hier erstmals systematisch neben den durchaus zahlreichen Forschungsbüchern über den Nationalsozialismus auch und vor allem die Erkenntnisse des sehr umfangreichen Materials der Nürnberger Prozesse.

58 Die Äußerung von John Herz in Bezug auf die persönliche Motivation, sich im Zweiten Weltkrieg im Kampf gegen das totalitäre deutsche Herrschaftsregime im OSS-Apparat zu engagieren, steht pars pro toto für die emigrierten jüdischen Intellektuellen aus Deutschland. Herz sprach in einem Interview mit Söllner davon, dass es »doch eine Sache von weltgeschichtlicher Bedeutung [war], den Faschismus niederzuringen und die demokratischen Systeme zu verteidigen«. Herz weiter: »lch verspürte die moralische Pflicht, gerade als Emigrant, der von Amerika aufgenommen worden war, etwas zu tun, um die Weltherrschaft des Faschismus zu verhindern.«(Söllner, Zur Archäologie der Demokratie in Deutschland, 1, S. 44 f.) 
Aufgrund einer militärischen Anweisung von Roosevelt wurde am 13. Juni 1942 das bereits angesprochene OSS gegründet. Das OSS sollte als die zentrale amerikanische Nachrichten- und Geheimdienstbehörde die militärische Entwicklung des Zweiten Weltkrieges aus der Sicht der US-Planer und -Strategen im Konnex mit anderen Regierungsbehörden von ganz entscheidender Bedeutung sein und darum die den kriegsbedingten Erfordernissen nicht mehr ausreichende alte Behörde COI ersetzen. Denn: Das zentrale Merkmal des OSS nach dieser Reorganisation war die "hochgradige Verzahnung von Informationsbeschaffung und -auswertung, strategischer Planung und subversiven Operationen - im OSS waren die für die Produktion von intelligence zuständigen nachrichtendienstlichen Sektionen mit den in der psychologischen Kriegführung und der Sabotage agierenden Operationseinheiten vereint $\star^{59}$.

Gleichwohl wurde als ein ganz entscheidender Arbeitsschwerpunkt die Sammlung und Analyse strategisch wichtiger Nachrichten für die militärische Führungsspitze der US-Streitkräfte, der Joint Chiefs of Staff (JCS), fokussiert. Innerhalb der gigantischen Kriegsadministration der Vereinigten Staaten war das OSS in den innenund außenpolitisch im Kriegsverlauf mitverantwortlichen Institutionen der explizit für den Zweiten Weltkrieg konstituierten JCS direkt zugeordnet. Hierbei bekamen die Forschergruppen in der R\&A - ebenjene Abteilung ist im Folgenden von zentraler Bedeutung - unter der Überwachung von sogenannten editors wie zum Beispiel dem damals sehr jungen Historiker Arthur M. Schlesinger jr. ${ }^{60}$ die Aufgabe übertragen, Informationen zu liefern

für die Planung und Ausführung von strategic services operations, d. h. für Aktionen der irregulären Kriegführung zur Schwächung des gegnerischen Kampfwillens sowie zur Unterstützung militärischer Operationen. Zum anderen waren die R\&A-Abteilungen dafür zuständig, auf Anfrage der JCS, der Streitkräfte und anderer autorisierter Regierungsbehörden nachrichtendienstliches Wissen bereitzustellen. Konkret bedeutete das die Sammlung, Auswertung und Analyse politischer, psychologischer, soziologischer, ökonomischer, topographischer und militärischer Daten sowie die Ausarbeitung entsprechender Studien, Karten und Graphiken. ${ }^{61}$

In der Washingtoner Zentrale der Organisation des OSS - organisiert in insgesamt fünf Hauptabteilungen, von denen die R\&A-Abteilung nur eine war, die gleichwohl als sozusagen intellektuelle, theoretische Schaltzentrale des riesigen Geheimdienstapparates operierte - arbeiteten unter der Leitung der Führung Donovans und dessen

59 Stefanie Middendorf, »Verstoßenes Wissen«. Emigranten als Deutschlandexperten im Office of Strategic Services und im amerikanischen Außenministerium 1943-1955, in: Neue Politische Literatur 46 (2001), S. 23-52, hier S. 26.

60 Schlesinger jr. wurde 1917 in Columbus (Ohio) geboren. Er studierte Geschichte an der Harvard-Universität in Boston, wurde Mitglied der Society of Fellows von 1939 bis 1942 in Harvard und arbeitete von 1942 bis 1943 im Kriegsinformationsamt; danach fungierte er als Nachrichtenoffizier und politischer Berater zwischen 1943 und 1945 im OSS, zuerst in Washington, dann in London, Paris und abschließend in Deutschland. Nach dem Zweiten Weltkrieg sollte er zu einem der bekanntesten Historiker und politisch einflussreichsten Publizisten nicht nur in den Vereinigten Staaten aufsteigen. Schlesinger zählte auch zu dem Autorenkreis der Zeitschrift Der Monat, die u. a. Auszüge aus seinem 1949 erschienenen The Vital Center veröffentlichte. Auf dieses Buch komme ich in Kapitel IV.3 zurück.

61 Middendorf, »Verstoßenes Wissen«, S. 26. 
offiziell als Sonderassistent firmierenden Allen W. Dulles (überwachte alle OSS-Operationen in Europa) in den Jahren des Zweiten Weltkrieges bis zu 13000 Angestellte, davon allein in der R\&A zeitweise über 1000 Mitarbeiter. Unter dem »Teamchef« Langer sollten hier die grundsätzlichen Analysen für die politischen Entscheidungsträger der amerikanischen Kriegsbürokratie insgesamt und für die über 40 Abteilungen innerhalb der OSS-Administration angefertigt werden. In den nach regionalen Kriterien aufgebauten vier R\&A-Divisionen Europa-Afrika, UdSSR, Fernost und Lateinamerika waren unter anderem die erwähnten hochkarätigen amerikanischen und internationalen Akademiker beschäftigt, die neben der Analyse des nationalsozialistischen Herrschaftsregimes sowie Planungen über die Deutschlandpolitik nach Beendigung des Zweiten Weltkrieges sich auch und vor allem frühzeitig Gedanken machten und anspruchsvolle Arbeiten anfertigten über die von den Vereinigten Staaten betriebene komplexe, nicht allein auf den europäischen Kontinent bezogene Nachkriegspolitik. Im Zusammenhang mit der Politik der Vereinigten Staaten im Kontext des Kalten Krieges ist hier von zentraler Bedeutung, dass selbst in der Hochphase der militärischen Konfrontation mit dem nationalsozialistischen Gewaltsystem in der R\&A-Division UdSSR die politikstrategisch und wissenschaftliche Auseinandersetzung mit dem kommunistischen Staat unter Stalin weiterging. Dabei ist zu berücksichtigen, dass in den USA bereits seit den 1920er-Jahren die politische Auseinandersetzung mit der Sowjetunion auf intellektuelle und wissenschaftliche Beine gestellt worden war und hierbei mit Samuel Harper einer der renommiertesten Sowjetforscher des Landes gewonnen werden konnte. Des Weiteren existierte mit der sogenannten Russlandabteilung des State Department eine Gruppe von Sowjetexperten, die die Analyse mit dem russischen Kommunismus mit wissenschaftlichen Methoden betrieben. Wie für die R\&A insgesamt galt auch für die Regionalabteilung UdSSR, dass hier eine qualitativ bemerkenswerte Verquickung von Geheimdienstagentur und einer großen Beteiligung von intellektuellen Mitarbeitern bestand, für die eine politische, wissenschaftliche und primär analytisch ausgerichtete Forschung ihres Untersuchungsgegenstandes im Zentrum ihres OSS-Engagements stand.

\subsection{Die "Kommunismusforschung « in der US-Administration nach dem Sieg gegen den Nationalsozialismus}

Der Sieg der alliierten Truppen über den nationalsozialistischen Staat und die Kapitulation der deutschen Wehrmacht am 8. Mai 1945 bedeutete gleichfalls das Ende der R\&A als Abteilung des kriegsbedingten und -wichtigen OSS. Denn die Beendigung der kriegerischen Auseinandersetzungen führte zur Auflösung des donovanschen Geheimdienst- und Nachrichtenapparates im September 1945, was allerdings nicht gleichbedeutend war mit der restlosen Auflösung der Forschungsabteilung. Anlass war eine Verfügung des neuen US-Präsidenten Harry Spencer Truman, der noch während des Zweiten Weltkrieges die Führungsposition des Staates von dem am 12. April 1945 verstorbenen Roosevelt übernahm. Die Verfügung im September 1945 zählte zu einer der ersten Amtshandlungen von Truman. Während die Mitarbeiter der traditionellen, d.h. der genuin geheimdienstlichen Abteilungen zum War Department hinüberwechselten, erfolgte im Rahmen des Abbaus der riesengroßen Washingtoner Kriegsbürokratie die Übernahme der Forschungsabteilung R\&A durch das State De- 